REVISTA MATEMATICA de la

Universidad Complutense de Madrid.

Volumen 2, sumero suplementario, 1989.

http://dx.doi.org/10.5209/rev_REMA.1989.v2.18106

\title{
Some normability conditions on Fréchet spaces
}

\author{
TOSUN TERzIOǴLU and DIETMAR VOGT
}

ABSTRACT. We define two new normability condition on Fréchet spaces and announce some related results.

The purpose of this note is to announce some results related to two normability conditions on Fréchet spaces, which will be given subsequently. Results stated here and their proofs are contained in [5] and [6].

We use the standard terminology and notation of the theory of locally convex spaces as in [3]. By $\left(\|\|_{k}\right)$ we always denote an increasing sequence of seminorms defining the topology of a Fréchet space $E$ and $U_{k}=\left\{x \in E:\|x\|_{k} \leqslant I\right\}$. $A=\left(a_{t, k}\right)$ stands for an infinite matrix of real numbers which always satisfies

$$
0 \leqq a_{j, k} \leqq a_{j: k+1} \text { and } \sup _{k} a_{j, k}>0
$$

for all $j$ and $k$. For $I \leqq p<+\infty$ we set

$$
\lambda^{\rho}(A)=\left\{\xi=(\xi): i\left|\xi \|_{k}^{p}=\Sigma\right| \xi ; j a_{j, k}^{p}<+\infty \text { for all } k\right\} .
$$

Equipped with seminorms \|\|$_{k^{\prime}} k=1,2, \ldots, \lambda(A)$ is a Fréchet space. For $\lambda^{\prime}(A)$ we simply write $\lambda(A)$.

We call a Fréchet space $E[\tau]$ locally normable [5] if there is a continuous norm $\|f\|$ on $E$ such that on every bounded subset $t$ coincides with the topology defined by this norm \|\| . This means that if $\left(x_{n}\right)$ is a bounded sequence in $E$ and $\lim \left\|x_{n}\right\|=0$ then we have $\lim x_{n}=0$.

For a locally normable Fréchet space $E$ we may assume without loss of generality that $] ! \|_{1}$ is the norm specified in the definition. So for every bounded subset $B$ and $k$ there is a $\varepsilon>0$ with

$$
\left(\varepsilon U_{1}\right) \cap B \subset U_{k^{*}}
$$

1980 Mathematics Subject Classification (1985 revision): 46 A06 Editorial de la Universidad Complutense. Madrid, 1989. 
Upon polarization we obtain

$$
U_{k}^{0} \subset E^{\top}\left[U_{1}^{0}\right]+B^{\circ}
$$

where $E\left[U_{1}^{0}\right]$ is the subspace of the dual $E^{\prime}$ spanned by $U_{1}^{0}$. Hence in this case

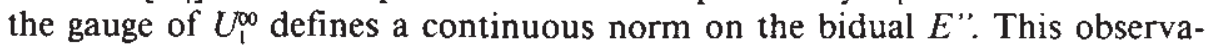
tion yields our first result.

(1) The bidual of a locally normable Fréchet space admits a continuous norm.

In the case of Köthe spaces we can characterize local normability in terms of the defining matrix.

(2) The following are equivalent:

(i) $\lambda^{P}(A)$ is locally normable for some $1 \leqq p<+\infty$

(ii) $\lambda^{p}(A)$ is locally normable for all $1 \leqq p<+\infty$

(iii) A satisfies the following condition:

(*) there is a $k_{0}$ such that for any choice of $C_{n}>0$ and for any $k$ there is a $C>0$ so that

$$
a_{j: k} \leqq \max \left\{C a_{j: x_{0},} \quad \sup _{n} \frac{a_{j, n}}{C_{n}}\right\}
$$

holds for all $j$.

The case $p=l$ is of special importance.

(3) $\lambda(A)$ is locally normable if and only if the bidual $\lambda(A)$ " admits a continuous norm.

Dierolf and Moscatelli [1] gave an example of a Fréchet space whose topology is defined by a sequence of norms but whose bidual admits no continuous norm. Using (3) and the condition $(*)$, it is easy to construct examples of Kothe spaces which exhibit the same phenomenon. In fact the well-known Grothendieck-Köthe example ([3];\$31,7) of a non-distinguished Fréchet space is a Köthe space $\lambda(A)$ which admits a continuous norm, but since $\mathrm{A}$ violates $\left({ }^{*}\right)$, the bidual $\lambda(A)$ " does not admit a continuous norm. For this matrix $A$, $\lambda^{2}(A)$ is a reflexive Fréchet space with a continuous norm, but it is not locally normable by (2). Hence the converse of (1) is false in general. Let us remark that one can also construct a quasinormable Köthe space which admits a continuous norm but whose bidual again has no continuous norm [5].

Let $\phi:(0, \infty) \rightarrow(0, \infty)$ be a strictly increasing function. We say $E$ satisfies $\left(D N_{\phi}\right)$ if we have a $k_{0}$ with the property that for each $k$ there is a $p$ and $C>0$ so that 


$$
\|x\|_{k} \leqq C \phi(r)\|x\|_{k_{0}}+\frac{l}{r}\|x\|_{p}
$$

holds for every $x \in E$ and $r>0$ ([8], [10]). We call a Fréchet space asymptotically normable [6] if it satisfies $\left(\mathrm{DN}_{\theta}\right)$ for some function $\phi$. For the role played by asymptotically normable Fréchet spaces in the vanishing of the functor Ext we refer to [10] and [12].

If a Fréchet space $E$ is a asympotically normable then for $k \geqq k_{0}$ on the ball $U_{p}\|\|_{k}$ and \|\|$_{k}$ define equivalent topologies, where $k_{0}, k$ and $p$ are as in the definition given above. In particular, if $\left(x_{n}\right)$ is Cauchy with respect to \|\|$_{p}$ and $\lim \left\|x_{n}\right\|_{k_{\theta}}=0$ then lim $\left\|x_{n}\right\|_{k}=0$. From this we obtain

(4) An asymptotically normable Fréchet is locally normable and also countably normable.

It is known that a countably normable Fréchet-Schwartz space is asymptotically normable [10]. It is also easy to show that a Fréchet-Montel space which admits a continuous norm is locally normable. However, even a nuclear Fréchet space which admits a continuous norm need not be asymptotically normable, a fact which was used in constructing nuclear Fréchet spaces without the bounded approximation property [2], [9].

One can characterize asymptotical normability of a Köthe space $\lambda(A)$ in terms of the defining matrix and also construct a Köthe-Montel space which admits a continuous norm but which is not asympotically normable [6]. Hence a locally normable Fréchet space need not be asymptotically normable.

We note that for $\phi(r)=r$, the condition $\left(\mathrm{DN}_{\oplus}\right)$, denoted by (DN), was introduced already in [7] to characterize the subspaces of the space (s) of rapidly decreasing sequences. Subsequently in [11] it was proved that every Fréchet space of type (DN) is isomorphic to a subspace of $l_{\infty}(I) \hat{\otimes}_{\mathrm{n}}(s)$ for some index set $\mathrm{I}$. As a generalization of this result we have

(5) For every increasing function $\phi:(0, \infty) \rightarrow(0, \infty)$ there is a nuclear Kothe space $\lambda(A)$ with a continuous norm such that every Fréchet space which satisfies $\left(D N_{\phi}\right)$ is isomorphic to a subspace of $l_{\infty}(I) \hat{\otimes}_{\pi} \lambda(A)$ for some index set $I$.

This means that the class of asymptotically normable Fréchet spaces is precisely the class of all Fréchet spaces which contains all Banach spaces as well as all nuclear Köthe spaces admitting continuous norms and which is closed under the operations of taking complete projective tensor products and passing to subspaces. The parallelism between this and the characterization of the quasinormable Fréchet spaces given by Meise and Vogt [4] is worth noting. 


\section{References}

[1] S. Dierolf and V.B. Moscatelli: A Fréchet space which has a continuous norm but whose bidual does not. Math. Z. 191, 17-21 (1986).

[2] ED DubINSKY: Nuclear Fréchet spaces without the bounded approximation property. Studia Math. 71, 85-105 (1981).

[3] G. KörHE: Topological Vector Spaces I, II. New York-Heidelberg-Berlin, 1969 and 1979.

[4] R. MEISE and D. VOGT: A characterization of the quasi-normable Frechet spaces. Math. Nachr. 122, 141-150 (1985).

[5] T. TERZIoĞLU and D. VOGT: A Köthe space which has a continuous norm but whose bidual does not. to appear in Arch. Math.

[6] T. TERZIOǴLU and D. VOGT: On asymptotically normable Fréchet spaces. Preprint.

[7] D. VoGT: Charakterisierung der Unterräume von s. Math. Z. 155 , 109-117 (1977).

[8] D. VoGT: Subspaces and quotient spaces of (s); in Functional Analysis: Surveys and Recent Results. K.D. Bierstedt and B. Fuchssteiner (eds.), North-Holland Math. Studies 27, 167-187, 1977.

[9] D. VoGT: An example of a nuclear Fréchet space without the bounded approximation property. Math. Z. 182, 265-267 (1983).

[10] D. VoGT: Some results on continuous linear maps between Fréchet spaces, in: Functional Analysis: Surveys and Recent Result Results III, K.D. Bierstedt and B. Fuchssteiner (eds.), North-Holland Math. Studies $90,349-381,1984$.

[11] D. VOGT: On two classes of $(F)$-spaces. Arch. Math. 45, 255-266 (1985).

[12] D. VoGT: On the functors $E x t^{\prime}(E, F)$ for Fréchet spaces. Studia Math. 85, 163-197 (1987).

Mathematics Department

Middle East Technical University

06531 Ankara-TURKEY
Fachbereich Mathematik

Bergische Universität GHS Wuppertal Gaußstr. 20 D-5600 Wuppertal 1 Fed. Rep. Germany 